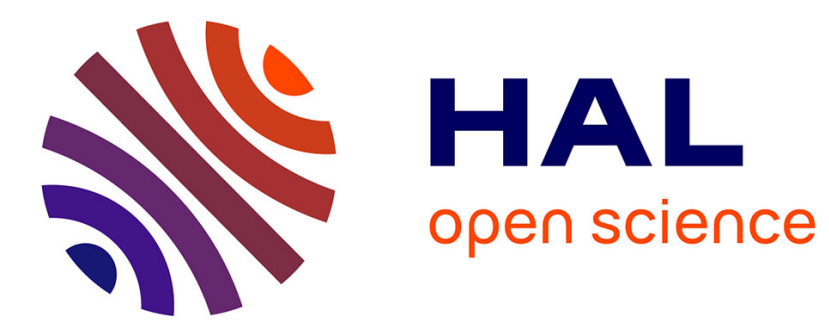

\title{
Kalman Filter-Based Channel Estimation for Mobile-to-Mobile and Relay Networks
}

\author{
Ali Houssam El Husseini, Laurent Ros, Eric Pierre Simon
}

\section{To cite this version:}

Ali Houssam El Husseini, Laurent Ros, Eric Pierre Simon. Kalman Filter-Based Channel Estimation for Mobile-to-Mobile and Relay Networks. IEEE Signal Processing Letters, 2019, 26 (5), pp.680-684. 10.1109/LSP.2019.2904439 . hal-02122959

\section{HAL Id: hal-02122959 \\ https://hal.science/hal-02122959}

Submitted on 7 May 2019

HAL is a multi-disciplinary open access archive for the deposit and dissemination of scientific research documents, whether they are published or not. The documents may come from teaching and research institutions in France or abroad, or from public or private research centers.
L'archive ouverte pluridisciplinaire $\mathbf{H A L}$, est destinée au dépôt et à la diffusion de documents scientifiques de niveau recherche, publiés ou non, émanant des établissements d'enseignement et de recherche français ou étrangers, des laboratoires publics ou privés. 


\title{
Kalman filter-based channel estimation for mobile-to-mobile and relay networks
}

\author{
Ali Houssam El Husseini ${ }^{1}$, Laurent Ros ${ }^{2}$, Eric Pierre Simon ${ }^{1}$,
}

\begin{abstract}
This paper deals with channel estimation in mobileto-mobile communications, possibly including amplify and forward mobile relays, assuming a two-dimensional scattering environment. A second-order autoregressive (AR(2)) model with the Kalman filter is used to estimate the channel gain of the global multi-hop channel. The parameters of the AR(2) model can be tuned by using a classic correlation matching criterion. Alternatively, the use of a minimization of the asymptotic variance criterion permits a strong performance improvement, although the corresponding analytical study is still missing in the literature-which is the topic of this paper. The closed-form expressions for the optimal tuning of the $\operatorname{AR}(2)$ parameters and mean square error (MSE) performance have been first established as a function of the second and fourth moments of the Doppler spectrum of the global channel. Because the analytical formulas of these moments were not given in the literature, we derive them by exploiting cumulant property of convolution of density functions, after decomposition of the global channel as a cascade of Jakes Doppler power spectral density channels. Consequently, the provided closed-form formula are helpful for the design of Kalman filters for a given state of the source, destination and mobile relays (Doppler spread and signal to noise ratio (SNR)).
\end{abstract}

Index Terms - channel estimation, autoregressive model, Jakes' Doppler, Kalman filter, mobile-to-mobile, relay networks.

\section{INTRODUCTION}

The study of wireless mobile-to-mobile (M-M) communications, possibly with amplify and forward relays, has received considerable attention. This type of communication enables the development of new applications, especially for vehicleto-vehicle systems and cooperative networks, where a direct communication link between mobile stations often holds without the intervention of fixed and highly elevated base stations. In such scenarios, the radio range can be extended via mobile terminals which act as relays. Adequate channel estimation is required for the development, analysis, and optimization of M-M and mobile relay network communication systems. Kalman filters (KFs) [1]-[3] are commonly used to track time varying complex gains (CG) of such channels. The design of a $\mathrm{KF}$ requires a linear recursive state-space representation of the parameter to be observed. The most used approximation model, especially for channel estimation problems, is the auto regressive model with order $p, \operatorname{AR}(p)$, where the parameters are tuned by matching the autocorrelation of the

A.H. EL Husseini and E.P. Simon are with the University of Lille and IEMN lab, Villeneuve d'Ascq, France. E.P. Simon is also a member of IRCICA. emails: ali.el-husseini@univ-lille.fr, eric.simon@univ-lille.fr.

L. Ros is with the Univ. Grenoble Alpes, CNRS, Institute of Engineering, Grenoble-INP, GIPSA-lab, 38000, Grenoble, France. E-mail: laurent.ros@gipsa-lab.grenoble-inp.fr true channel CG with that of the AR process. This is known as the correlation matching (CM) criterion [4]-[8]. The CM criterion is simple to implement and adequate for fast fading channels. However, for a slow fading scenario (normalized Doppler frequency less than $10^{-2}$ ), which is valid for usual vehicular communications, using the $\mathrm{CM}$ criterion gives poor results compared to the minimum asymptotic variance (MAV) criterion, as seen in [9]-[11] for AR with order $p=1$, and in [12] for Fix-to-Mobile channel.

The main contribution of this paper is to provide analytic expressions for the parameters of a KF based on secondorder AR model (AR(2)) tuned under MAV criterion, together with the corresponding steady-state MSE performance, for $\mathrm{M}-\mathrm{M}$ and mobile relays channels. These formulas are first derived with respect to the Doppler spectrum moments in Section II. The expressions of these moments are established in Section III by using cumulant property of convolution of density functions. Section IV validates the analysis by means of MSE simulations, the first and second order AR model-based KFs (combined with CM and MAV criterion) are selected as references.

\section{SYSTEM MODEL AND FRAMEWORK}

\section{A. Observation model}

Let us consider a communication channel with possible relays between a source $\mathrm{S}$ and a destination $\mathrm{D}$. The discretetime observation equation $y_{(k)}$ at $\mathrm{D}$ is:

$$
y_{(k)}=\alpha_{(k)}+w_{(k)}{ }^{1}
$$

where $\alpha_{(k)}$ is the channel complex gain between $\mathrm{S}$ and D and $w_{(k)}$ is the complex circular white observation noise with variance $\sigma_{w}^{2}$. The parameter to be estimated $\alpha$ is assumed to be a zero-mean circular complex narrow-band stationary process with variance $\sigma_{\alpha}^{2}$, with a power spectrum density (PSD) $\Gamma(f)$ with a maximum Doppler frequency $f_{\max }$. The $n$th order moment $\mu_{(n)}$ of $\Gamma(f)$ will play an important role in the estimation performance. It is defined by:

$$
\mu_{(n)}=(2 \pi T)^{n} \int_{-\infty}^{+\infty} f^{n} \Gamma(f) d f,
$$

\footnotetext{
1 This model comes from a flat fading communication and is obtained after normalizing the received signal by the constant modulus pilot symbol. Although it is admittedly simplistic, it can be applied in more involved contexts, such as pilot-aided multi-carrier systems in frequency-selective wireless channels.
} 
and $\tilde{\mu}_{(n)}=\frac{\mu_{(n)}}{\sigma^{2}}$ denotes their normalized version. The Doppler spread $B_{d}$ is defined as the square root of the normalized second order moment of $\Gamma(f)$ [13, Eq. (16)]:

$$
B_{d}=\frac{1}{2 \pi T} \sqrt{\tilde{\mu}_{(2)}}
$$

\section{B. State model and Kalman filter}

The true channel CG $\alpha$ is approximated by second order autoregressive model $\operatorname{AR}(2)$ denoted by $\tilde{\alpha}$ :

$$
\tilde{\alpha}_{(k)}=a_{1} \tilde{\alpha}_{(k-1)}+a_{2} \tilde{\alpha}_{(k-2)}+u_{(k)},
$$

with $a_{1}=2 r \cos \left(2 \pi f_{\mathrm{AR}(2)} T\right)$ and $a_{2}=-r^{2}$, where $r \in[0,1[$ is the radius of the poles, and $f_{\mathrm{AR}(2)} T$ is the normalized resonance frequency of the $\mathrm{AR}(2)$ process. $u_{(k)}$ is the white circular complex Gaussian state noise with variance $\sigma_{u}^{2}$ calculated as $\sigma_{u}^{2}=\sigma_{\alpha}^{2}\left(1+a_{2}\right)\left(1-a_{1}-a_{2}\right)\left(1+a_{1}-a_{2}\right) /\left(1-a_{2}\right)$.

The second order autoregressive model can be reformulated as a state model. The state vector to be considered includes the channel CG at $k$ and $k-1, \boldsymbol{\alpha}_{(k)}=\left[\alpha_{(k)}, \alpha_{(k-1)}\right]^{T}$ and $\tilde{\boldsymbol{\alpha}}_{(k)}=\left[\tilde{\alpha}_{(k)}, \tilde{\alpha}_{(k-1)}\right]^{T}$. The state transition matrix is $\mathbf{M}=$ $\left[\begin{array}{cc}a_{1} & a_{2} \\ 1 & 0\end{array}\right]$ and the state noise vector is $\mathbf{u}_{(k)}=\left[u_{(k)}, 0\right]^{T}$. The selection vector of size $1 \times 2$ is $\mathbf{s}^{T}=[1,0]$. The state evolution (4) and observation (1) are then:

$$
\begin{aligned}
\tilde{\boldsymbol{\alpha}}_{(k)} & =\mathbf{M} \tilde{\boldsymbol{\alpha}}_{(k-1)}+\mathbf{u}_{(k)} \\
y_{(k)} & =\mathbf{s}^{T} \boldsymbol{\alpha}_{(k)}+w_{(k)} .
\end{aligned}
$$

from which the KF equations can be established as in [12] with the two-dimension Kalman gain vector $\mathbf{K}_{(k)}=\left[\begin{array}{l}K_{1(k)} \\ K_{2(k)}\end{array}\right]$ to produce the estimate vector $\hat{\boldsymbol{\alpha}}_{(k \mid k)}=\left[\hat{\alpha}_{(k \mid k)}, \hat{\alpha}_{(k-1 \mid k)}\right]^{T}$.

\section{Preliminary results}

In this section, the approach of [12] that was restricted to the Jakes' Doppler spectrum corresponding to the fix-to-mobile channel is extended to a more general band-limited Doppler spectrum $\Gamma_{\alpha}(f)$, possibly including relays. This has been done by rewriting the results as a function of the fourth moment $\mu_{(4)}$. Recall that the following assumptions are considered: slow fading $\left(f_{\max } T \ll 1, f_{\mathrm{AR}(2)} T \ll 1\right)$, SNR greater than $1\left(\sigma_{\alpha} \geq \sigma_{w}\right), r$ close to one, speed range of the KF slow enough to filter the measurement noise but sufficiently fast to track the fading process $\left(\left(2 \pi f_{\mathrm{AR}(2)} T\right)^{2} \ll \frac{\sigma_{u}}{\sigma_{w}} \ll 1\right)^{2}$. Under these assumptions, the following expression obtained in [14],

$$
r \simeq 1-\frac{\sigma_{u}^{2}}{4 \sigma_{\alpha}^{2}\left(2 \pi f_{\mathrm{AR}(2)} T\right)^{2}},
$$

shows that for a given $f_{\mathrm{AR}(2)}$, the $\mathrm{KF}$ can be tuned from $\sigma_{u}$ instead of $r$, as will be done. In this paper, $f_{\mathrm{AR}(2)}$ is fixed to be equal to the Doppler spread $B_{d}$ (3):

$$
f_{\mathrm{AR}(2)} T=B_{d} T=\frac{1}{2 \pi} \sqrt{\tilde{\mu}_{(2)}}
$$

In the following, we consider the steady state mode; i.e., after convergence of the KF to a time invariant filter. This

\footnotetext{
${ }^{2}$ Note that all these assumptions match the context of channel estimation in slow fading scenario and are widely accepted in the literature.
}

filter receives the observation $y$ as an input and gives the $\mathrm{CG}$ estimation $\hat{\alpha}$ as an output. Its transfer function $L(z)$ is [12]:

$$
L(z)=\frac{K_{1}+a_{2} K_{2} z^{-1}}{1+z^{-1}\left(a_{2} K_{2}-a_{1}\left(1-K_{1}\right)\right)-a_{2}\left(1-K_{1}\right) z^{-2}},
$$

where the steady state Kalman gains are given by:

$$
K_{1} \simeq \sqrt{2 \frac{\sigma_{u}}{\sigma_{w}}} \quad \text { and } \quad K_{2}=\frac{a_{1}\left(1-K_{1}\right) K_{1}}{1-a_{2}+a_{2} K_{1}} .
$$

The MSE, defined by $\sigma_{\epsilon}^{2}=E\left\{\left|\alpha_{(k)}-\hat{\alpha}_{(k \mid k)}\right|^{2}\right\}$ when $k \rightarrow$ $+\infty$, can be decomposed as follows: $\sigma_{\epsilon}^{2}=\sigma_{\epsilon w}^{2}+\sigma_{\epsilon \alpha}^{2}$.

- The static error variance $\sigma_{\epsilon w}^{2}$ is due to the additive noise $w_{(k)}$ filtered by the low pass filter $L(z)$ [12]:

$$
\sigma_{\epsilon w}^{2} \stackrel{\text { def }}{=} \sigma_{w}^{2} T \int_{-\frac{1}{2 T}}^{\frac{1}{2 T}}\left|L\left(e^{2 i \pi f T}\right)\right|^{2} d f \simeq \sigma_{w}^{2} \frac{3 K_{1}}{4}
$$

- The dynamic error variance $\sigma_{\epsilon \alpha}^{2}$ is due to the variations of $\alpha_{(k)}$ filtered by the high pass filter $1-L(z)$ :

$\sigma_{\epsilon \alpha}^{2} \stackrel{\text { def }}{=} \int_{-f_{\max }}^{+f_{\max }}\left|1-L\left(e^{2 i \pi f T}\right)\right|^{2} \Gamma(f) d f \simeq \int_{-f_{\max }}^{+f_{\max }} \frac{(2 \pi f T)^{4}}{K_{1}^{4}} \Gamma(f) d f$,

From (12), it is deduced that the resultant expression is the 4th moment of the PSD divided by $K_{1}^{4}$. Finally,

$$
\sigma_{\epsilon}^{2}=\sigma_{w}^{2} \frac{3 K_{1}}{4}+\frac{\mu_{(4)}}{K_{1}^{4}}
$$

where $K_{1}$ in terms of $\sigma_{u}$ and $\sigma_{w}$ (see Eq. (10)).

We can now obtain the minimization of $\sigma_{\epsilon}^{2}$. First, differentiating (13) with respect to $\sigma_{u}^{2}$ after substituting $K_{1}$ with its expression in (10) and equating to zero leads to:

$$
\sigma_{u(\mathrm{MAV})}^{2}=\left(\frac{8}{9} \mu_{(4)}^{2} \sigma_{w}\right)^{\frac{2}{5}}
$$

and then to the expression for the minimum MSE:

$$
\sigma_{\epsilon(\mathrm{MAV})}^{2}=\frac{5}{4}\left(\frac{8}{9} \sqrt{\mu_{(4)}}\left(\sigma_{w}^{2}\right)^{2}\right)^{\frac{2}{5}} .
$$

From (14), (7) and (8) we conclude that, for a given observation noise level $\sigma_{w}^{2}$, the tuning of the $\mathrm{AR}(2)-\mathrm{KF}$ only depends on the fourth moment $\mu_{(4)}$ and the second normalized moment $\tilde{\mu}_{(2)}=\left(2 \pi B_{d} T\right)^{2}$. With this tuning, the minimum MSE (15) relies only on $\mu_{(4)}$. To obtain optimal tuning and performance of the KF, the objectives of the next section is to evaluate $\mu_{(2)}$ and $\mu_{(4)}$. We will first recall the results for the fix-to-mobile channel (Jakes Doppler model), which is used as a reference, also denoted by partial link in the following sections, and we then establish new values of $\mu_{(2)}$ and $\mu_{(4)}$ for more complex channels including mobile relays. 


\section{COMMUNICATION CHANNELS AND MOMENTS CALCULATION}

\section{A. Fix-to-mobile communication channel ( partial link)}

Under scattering conditions, the Jakes' Doppler spectrum of the circular complex Gaussian CG, for fix-to-mobile communication (F-M), also called single Rayleigh link, is:

$$
\Gamma^{\mathrm{F}-\mathrm{M}}(f)=\sigma_{\alpha}^{2} \tilde{\Gamma}_{f_{x}}^{\mathrm{F}-\mathrm{M}}(f)= \begin{cases}\frac{\sigma_{\alpha}^{2}}{\pi f_{x} \sqrt{1-\left(\frac{f}{f_{x}}\right)^{2}}} & \text { if }|f|<f_{x} . \\ 0 & \text { if }|f|>f_{x} .\end{cases}
$$

where $f_{x}$ is the Doppler frequency and $\tilde{\Gamma}_{f_{x}}(f)$ is the normalized Jakes' Doppler PSD (with integral equals to one). The autocorrelation coefficient $R^{\mathrm{F}-\mathrm{M}}[m]$ of the stationary $\mathrm{CG} \alpha_{(k)}$ is defined for a lag $m$ by:

$$
R^{\mathrm{F}-\mathrm{M}}[m]=\mathrm{E}\left\{\alpha_{(k)} \cdot \alpha_{(k-m)}^{*}\right\}=\sigma_{\alpha}^{2} J_{0}\left(2 \pi f_{x} T m\right),
$$

where $J_{0}$ is the zeroth-order Bessel function of the first kind.

Invoking the moment property, the moments $\mu_{(n)}$ of the spectrum $\Gamma(f)$ of the CG defined in (2) can be obtained by:

$$
\mu_{(n)}=(j)^{-n} R^{(n)}(0),
$$

where $R(t)$ is the continuous-time autocorrelation function of the CG and $R^{(n)}(0)=\left.\frac{d^{n}}{d t^{n}} R(t)\right|_{t=0}$.

The second and fourth moments of the Jakes' Doppler spectrum in (16) are then calculated by differentiating (17) using (18), and the tuning frequency $f_{\mathrm{AR}(2)} T$ is deduced from (8):

$$
\begin{aligned}
\mu_{(2)}^{\mathrm{F}-\mathrm{M}} & =\sigma_{\alpha}^{2} \cdot \tilde{\mu}_{(2), f_{x}}^{\mathrm{F}-\mathrm{M}}=\frac{1}{2} \sigma_{\alpha}^{2}\left(2 \pi f_{x} T\right)^{2} \\
\mu_{(4)}^{\mathrm{F}-\mathrm{M}} & =\sigma_{\alpha}^{2} \cdot \tilde{\mu}_{(4), f_{x}}^{\mathrm{F}-\mathrm{M}}=\frac{3}{8} \sigma_{\alpha}^{2}\left(2 \pi f_{x} T\right)^{4} \\
f_{\mathrm{AR}(2)} T & =B_{d} T=\frac{f_{x} T}{\sqrt{2}}
\end{aligned}
$$

\section{B. Mobile-to-mobile communication channel}

In [15], Akki and Haber show that in isotropic scattering conditions around S and D, the M-M model is similar to two independent successive partial links. To be more precise, it is equivalent to a mobile-to-fix (M-F) and a fix-to-mobile (F-M) links with Doppler frequencies $f_{1}$ and $f_{2}$ due to the motions of $\mathrm{S}$ and $\mathrm{D}$, respectively. Hence, $\alpha=\alpha_{1} \alpha_{2}$, where $\alpha_{1}$ and $\alpha_{2}$ are independent zero-mean circular Gaussian complex variables with autocorrelation given in (17). Thus, autocorrelation of $\alpha$ is the product of two Bessel functions [9], [10], [15]:

$$
\begin{aligned}
R^{\mathrm{M}-\mathrm{M}}[m] & =\mathrm{E}\left\{\alpha_{1(k)} \alpha_{2(k)} \alpha_{1(k-m)}^{*} \alpha_{2(k-m)}^{*}\right\} \\
& =\sigma_{\alpha}^{2} J_{0}\left(2 \pi f_{1} T m\right) J_{0}\left(2 \pi f_{2} T m\right) .
\end{aligned}
$$

The PSD of $\alpha$ denoted by $\Gamma^{\mathrm{M}-\mathrm{M}}(f)$ is then the convolution of two partial Jakes PSD (16) with Doppler frequencies $f_{1}$ and $f_{2}$, respectively.

By differentiating the autocorrelation function in (22) using (18), the second and the fourth moment of the M-M communication are given in [16]:

$$
\begin{aligned}
& \mu_{(2)}^{\mathrm{M}-\mathrm{M}}=\frac{1}{2} \sigma_{\alpha}^{2}(2 \pi T)^{2}\left(f_{1}^{2}+f_{2}^{2}\right) \\
& \mu_{(4)}^{\mathrm{M}-\mathrm{M}}=\frac{3}{8} \sigma_{\alpha}^{2}(2 \pi T)^{4}\left(f_{1}^{4}+f_{2}^{4}+4 f_{1}^{2} f_{2}^{2}\right)
\end{aligned}
$$

According to (23) and (8), the normalized resonance frequency of the $\mathrm{AR}(2)$ process $f_{\mathrm{AR}(2)} T$ should be tuned to:

$$
f_{\mathrm{AR}(2)} T=B_{d} T=\sqrt{\frac{\left(f_{1} T\right)^{2}+\left(f_{2} T\right)^{2}}{2}}
$$

\section{Mobile relays channel}

For $N$ amplify and forward mobile relays between the mobile source and the mobile destination, and still assuming isotropic scattering conditions at each stage (allowing decomposition of each M-M link into two partial M-F and F-M links, as in the previous section), the global link $\mathrm{S}-\mathrm{D}$ is equivalent to $\mathrm{S}-\mathrm{F}, \mathrm{F}-\mathrm{R}_{1}, \mathrm{R}_{1}-\mathrm{F}, \ldots, \mathrm{F}-\mathrm{R}_{i}, \mathrm{R}_{i}-\mathrm{F}, \ldots ., \mathrm{F}-\mathrm{D}$, where $\mathrm{R}_{i}$ denotes the mobile relay, and $i \in[1, N]$. The global $\mathrm{CG} \alpha$ is the product of the zero-mean channel gains of the independent successive partial links. Then, the autocorrelation function of the resulting CG generalizes (22) with relays [10] as :

$$
R^{\text {relays }}[m]=\sigma_{\alpha}^{2} J_{0}\left(2 \pi f_{S} T m\right) \prod_{i=1}^{N}\left[J_{0}\left(2 \pi f_{i} T m\right)\right]^{2} J_{0}\left(2 \pi f_{D} T m\right),
$$

where $f_{S}, f_{i}, f_{D}$ are the Doppler frequency of the source, $i$ th relay and destination, respectively.

The Doppler PSD of the global CG is the Fourier transform of the autocorrelation function in (26), which is the convolution of all PSDs of the partial links between source and destination:

$$
\begin{aligned}
& \Gamma^{\text {relays }}(f)= \\
& \sigma_{\alpha}^{2} \tilde{\Gamma}_{f_{\mathrm{S}}}^{\mathrm{F}-\mathrm{M}}(f) \otimes \bigotimes_{i=1}^{N} \tilde{\Gamma}_{f_{i}}^{\mathrm{F}-\mathrm{M}}(f) \otimes \bigotimes_{i=1}^{N} \tilde{\Gamma}_{f_{i}}^{\mathrm{F}-\mathrm{M}}(f) \otimes \tilde{\Gamma}_{f_{\mathrm{D}}}^{\mathrm{F}-\mathrm{M}}(f),
\end{aligned}
$$

where $\otimes$ is the convolution operator.

This equation is valid for $|f| \leq\left|f_{\max }\right|$ where $f_{\max }=f_{S}+$ $2 \sum_{i=1}^{N} f_{i}+f_{D}$ is the spectrum support.

In the previous section, the moments $\mu_{(4)}$ and $\mu_{(2)}$ were calculated by differentiating the autocorrelation function and evaluating it at $t=0$. This method is intractable in the presence of $N$ relays. We suggest using another approach based on the following property ${ }^{3}$ : if a density function is the convolution of elementary density functions, then the cumulant of this function is the sum of the cumulant of each elementary function. By applying this property to the Doppler PSD of the $\mathrm{CG}$ in (27), we can conclude that the $n$th cumulant $K_{(n)}$ of the CG $\alpha$ is the sum of the cumulants of the CG of each partial link between the source and the destination:

$$
K_{(n)}^{\text {relays }}=\sigma_{\alpha}^{2}\left(\tilde{K}_{(n), f_{S}}^{\mathrm{F}-\mathrm{M}}+2 \sum_{i=1}^{N} \tilde{K}_{(n), f_{i}}^{\mathrm{F}-\mathrm{M}}+\tilde{K}_{(n), f_{D}}^{\mathrm{F}-\mathrm{M}}\right),
$$

where $\tilde{K}_{(n), f_{S}}^{\mathrm{F}-\mathrm{M}}, \tilde{K}_{(n), f_{i}}^{\mathrm{F}-\mathrm{M}}$ and $\tilde{K}_{(n), f_{D}}^{\mathrm{F}-\mathrm{M}}$ are the $n$th normalized cumulants for the PSDs of all the partial links between $\mathrm{S}$ and D. Having

$$
K_{(4)}=\mu_{(4)}-3 \mu_{(2)}^{2}, \quad \text { and } \quad K_{(2)}=\mu_{(2)}
$$

\footnotetext{
${ }^{3}$ This property corresponds to the well-known result in probability theory: the probability density function (PDF) of the sum of $N$ random independent variables results in the convolution of $N$ elementary PDFs, and the cumulant of this PDF is equal to the sum of the cumulants of the $N$ elementary PDFs.
} 
due to the fact that the CG is centered $\left(\mu_{(1)}=0\right)$, the second moment of relays channel is obtained from (28) for $n=2$ :

$$
\mu_{(2)}^{\text {relays }}=\sigma_{\alpha}^{2}\left(\tilde{\mu}_{(2), f_{S}}^{\mathrm{F}-\mathrm{M}}+2 \sum_{i=1}^{N} \tilde{\mu}_{(2), f_{i}}^{\mathrm{F}-\mathrm{M}}+\tilde{\mu}_{(2), f_{D}}^{\mathrm{F}-\mathrm{M}}\right) .
$$

And the fourth moment of relays channel, denoted by $\mu_{(4)}^{\text {relays }}$, is obtained by replacing the expressions of the cumulants in both sides of the equation in (28):

$$
\begin{aligned}
\mu_{(4)}^{\text {relays }} & =\sigma_{\alpha}^{2}\left(\tilde{\mu}_{(4), f_{S}}^{\mathrm{F}-\mathrm{M}}+\tilde{\mu}_{(4), f_{D}}^{\mathrm{F}-\mathrm{M}}+2 \sum_{i=1}^{N} \tilde{\mu}_{(4), f_{i}}^{\mathrm{F}-\mathrm{M}}\right. \\
& +3\left(\left(\tilde{\mu}_{(2), f_{S}}^{\mathrm{F}-\mathrm{M}}+\tilde{\mu}_{(2), f_{D}}^{\mathrm{F}-\mathrm{M}}+2 \sum_{i=1}^{N} \tilde{\mu}_{(2), f_{i}}^{\mathrm{F}-\mathrm{M}}\right)^{2}\right. \\
& \left.-\left(\tilde{\mu}_{(2), f_{S}}^{\mathrm{F}-\mathrm{M}}\right)^{2}-\left(\tilde{\mu}_{(2), f_{D}}^{\mathrm{F}-\mathrm{M}}\right)^{2}-2 \sum_{i=1}^{N}\left(\tilde{\mu}_{(2), f_{i}}^{\mathrm{F}-\mathrm{M}}\right)\right),
\end{aligned}
$$

The moments in Eq. (30) are given by (19) and (20). Note that M-M result (24) can be retrieved from the more general formula (30) by removing the $N$ relays.

The Doppler spread $B_{d}$ is still deduced from (3) and (29), which permits us to fix $f_{\mathrm{AR}(2)} T$ to:

$$
f_{\mathrm{AR}(2)} T=B_{d} T=\sqrt{\frac{\left(f_{S} T\right)^{2}+2 \sum_{i=1}^{N}\left(f_{i} T\right)^{2}+\left(f_{D} T\right)^{2}}{2}} .
$$

\section{Simulation RESUlTS AND CONCLUSION}

In this section, the Monte-Carlo simulations for M-M communications and mobile relays communications are considered. The channels are estimated with KF based on the AR(2) model tuned by our MAV criterion. For comparison, simulations with $\mathrm{AR}(2)$ tuned by the correlation matching $(\mathrm{CM})$ criterion of the literature are also shown. Fig. 1 plots the corresponding asymptotic MSE for $B_{d} T \in$ $\left\{10^{-2}, 10^{-3}, 10^{-4}\right\}$ and different values of SNR in the case of a mobile relays channel with $N=3$ relays. First, the theoretical values of the MSE with MAV (14) match well with the simulations for all the usual range of SNR between 0 and $20 \mathrm{~dB}$, which validates our approach. The MSE performance under MAV criterion is largely better than that obtained under $\mathrm{CM}$ criterion, especially for low $B_{d} T$. CM manifests diminished performance because the MSE is approximately constant with respect to $B_{d} T$ for a given SNR. This corroborates the result proved in [17, Eq. (22)] for AR(1) and F-M channels.

Fig. 2 compares the performance of the AR(2)-MAV to that of the AR(1)-MAV of the literature [9], [10] and the AR(2)$\mathrm{CM}$ for the case of M-M communications, with respect to the SNR, for $B_{d} T=10^{-4}$. It is seen that the KF with AR(2)-MAV outperforms the AR(1)-MAV and the AR(2)$\mathrm{CM}$, demonstrating the advantage of using an appropriate second order modeling versus a first order modeling. Again, the theoretical values (14) match the simulations.

To conclude, the problem of estimating a radio mobile channel including possible mobile relays channels in an isotropic

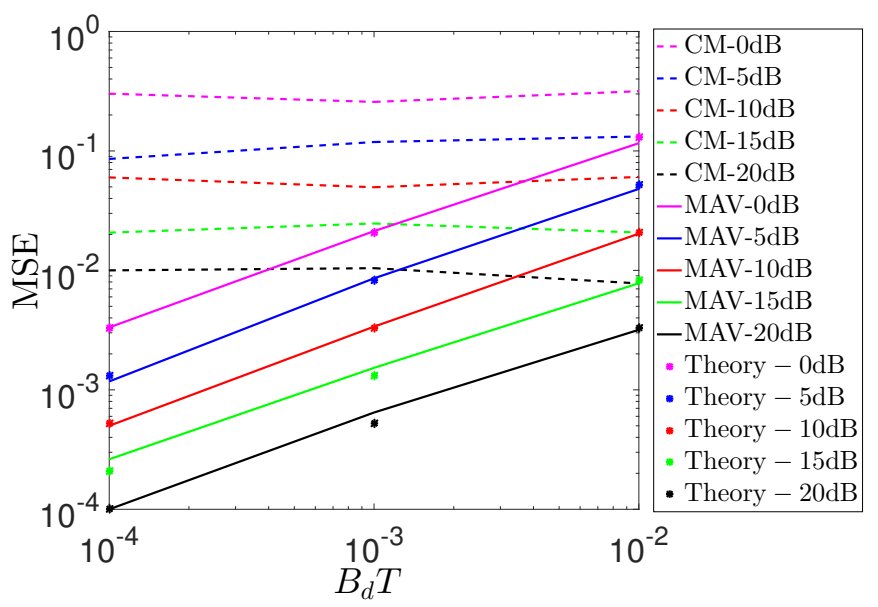

Fig. 1: Asymptotic MSE comparison of the proposed AR(2)-MAV with $\mathrm{AR}(2)-\mathrm{CM}$ and the theoretical MSE in (15) for different values of $B_{d} T$ and SNR with three AF mobile relays.

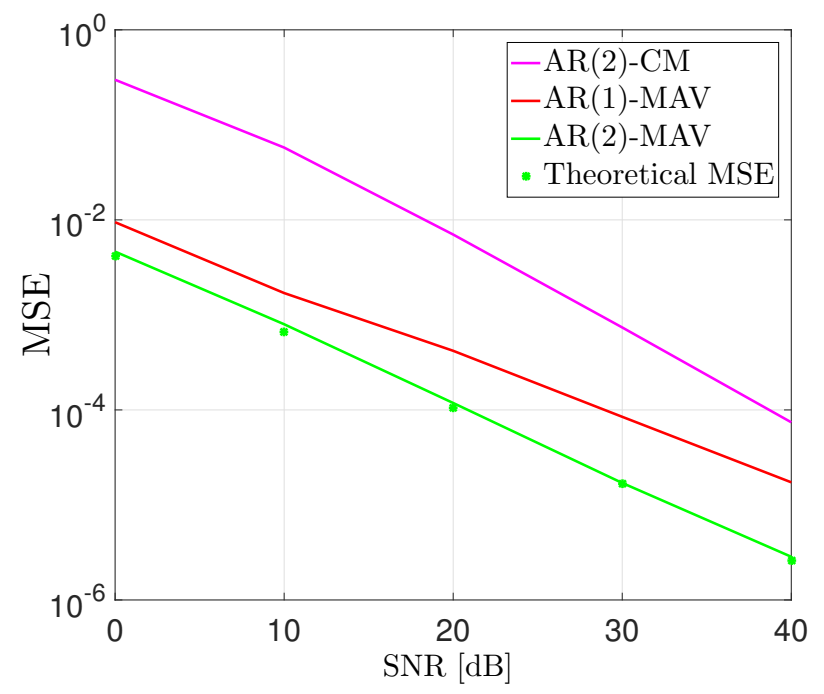

Fig. 2: Asymptotic MSE comparison of the proposed AR(2)-MAV with $\mathrm{AR}(2)-\mathrm{CM}$ and $\mathrm{AR}(1)-\mathrm{MAV}$ [10] for $B_{d} T=10^{-4}$ and different values of SNR for M-M communications.

two-dimensional scattering environment was addressed, assuming the use of a Kalman filter (KF) based on second order autoregressive model. First, the analytical relationships for the optimal tuning and performance of the $\operatorname{AR}(2)$ have been established. Moreover, we showed that they depend only on the second and fourth moments of the Doppler spectrum of the global channel. Second, using the moment and cumulant properties, these global moments have been expressed from the elementary moments of the partial Jakes' Doppler links that are known. Consequently, we provide performance and tuning formula in terms of the status of the source, the destination and the $N$ mobile relays (Doppler spread and SNR). The results of our simulation show the proposed AR(2) MAV approach outperforms the approaches of the literature based on $\mathrm{CM}$ criterion or $\mathrm{AR}(1)$ models. 


\section{REFERENCES}

[1] S. Locubiche-Serra, G. Seco-Granados, and J. A. López-Salcedo, "Closed-form approximation for the steady-state performance of secondorder kalman filters," IEEE Signal Process. Lett., vol. 25, no. 2, pp. 268-272, Feb 2018.

[2] — "Closed-form approximation for the convergence time ofpth-order kalman filters," IEEE Signal Process. Lett., vol. 25, no. 10, pp. 15051509, Oct 2018.

[3] R. Apelfrjd, W. Zirwas, and M. Sternad, "Joint reference signal design and kalman/wiener channel estimation for fdd massive mimo," 042018.

[4] K. E. Baddour and N. C. Beaulieu, "Autoregressive modeling for fading channel simulation," IEEE Trans. Wireless Commun, vol. 4, no. 4, pp. 1650-1662, July 2005.

[5] X. Zhou, T. A. Lamahewa, and P. Sadeghi, "Kalman filter-based channel estimation for amplify and forward relay communications," in 2009 Conference Record of the Forty-Third Asilomar Conference on Signals, Systems and Computers, Nov 2009, pp. 1498-1502.

[6] X. Shen, Y. Liao, X. Dai, M. Zhao, K. Liu, and D. Wang, "Joint channel estimation and decoding design for 5g-enabled v2v channel," China Communications, vol. 15, no. 7, pp. 39-46, July 2018.

[7] P. Lioliou, D. Svensson, and M. Viberg, "Channel tracking for af mimo relaying systems," in Vehicular Technology Conference (VTC Fall), 2012 IEEE. IEEE, 2012, pp. 1-5.

[8] K. J. Kim, R. A. Iltis, and H. V. Poor, "Frequency offset and channel estimation in cooperative relay networks," IEEE Transactions on Vehicular Technology, vol. 60, no. 7, pp. 3142-3155, Sep. 2011.

[9] S. Ghandour-Haidar, L. Ros, and J. M. Brossier, "3-d mobile-to-mobile channel tracking with first-order autoregressive model-based kalman filter," in IEEE International Symposium on Signal Processing and Information Technology, Dec 2013, pp. 000 464-000 469.

[10] S. Ghandour-Haidar, "Estimation de canal à évanouissements plats dans les transmissions sans fils à relais multibonds," Ph.D. dissertation, Université de Grenoble, 2014.

[11] S. Ghandour-Haidar, L. Ros, and J. Brossier, "Improving the tuning of first-order autoregressive model for the estimation of amplify and forward relay channel," in 2012 19th International Conference on Telecommunications (ICT), April 2012, pp. 1-6.

[12] A. H. El Husseini, E. P. Simon, and L. Ros, "Optimization of the second order autoregressive model AR(2) for Rayleigh-Jakes flat fading channel estimation with Kalman filter," in Digital Signal Processing. London, United Kingdom: IEEE, Aug. 2017.

[13] W. Dahech, M. Ptzold, C. A. Gutirrez, and N. Youssef, "A non-stationary mobile-to-mobile channel model allowing for velocity and trajectory variations of the mobile stations," IEEE Trans. Wireless Commun., vol. 16, no. 3, pp. 1987-2000, March 2017.

[14] A. H. El Husseini, L. Ros, and E. P. Simon, "Expression for the radius $r$ of an $\mathrm{AR}(2)$ process used to model a wireless communication channel," Dec. 2018, working paper or preprint. [Online]. Available: https://hal.archives-ouvertes.fr/hal-01952796

[15] A. S. Akki and F. Haber, "A statistical model of mobile-to-mobile land communication channel," IEEE Trans. Veh. Technol., vol. 35, no. 1, pp. $2-7,1986$

[16] A. S. Akki, "Statistical properties of mobile-to-mobile land communication channels," IEEE Trans. Veh. Technol., vol. 43, no. 4, pp. 826-831, Nov 1994.

[17] S. Ghandour-Haidar, L. Ros, and J.-M. Brossier, "On the use of firstorder autoregressive modeling for Rayleigh flat fading channel estimation with Kalman filter," Signal Processing, vol. 92, 2012. 